\title{
Clinical applications of proton MR spectroscopy in the diagnosis of brain tumours
}

\author{
Nail Bulakbasi \\ Gulhane Military Medical Academy, 06018 Etlik, Ankara, Turkey \\ Tel.: 90312304 4703; Fax: 90312326 0551; E-mail: nbulak@gata.edu.tr
}

\begin{abstract}
There are few but important problems in magnetic resonance (MR) diagnosis of the brain tumours such as predicting the grade, exact definition of the tumour borders, differentiation of the cystic tumours from abscess, the tumoral core from peritumoral oedema, and the tumour recurrence from radiation necrosis. MR spectroscopy (MRS) can add more information to MR imaging (MRI) in solving many of these problems. Widespread usage of faster MRS applications with higher signalto-noise ratio (SNR) and spatial resolution, allows us to detect functional metabolic changes, which provides more data to understand the exact nature of the tumour and the morphological and physiological changes occurring in the surrounding brain parenchyma.
\end{abstract}

\section{Introduction}

Since the introduction to clinical practice, MRI has provided new insights to the diagnosis of brain tumours. It has not only shown the existence of tumour, but also differentiated it form tumour-like lesions, such as the demyalinating, ischemic and even haemorrhagic lesions. Multi-planar imaging capability of MRI has also led to demonstrate the complications and the relationship of the tumour with the normal brain parenchyma. Although MRI has also had its own diagnostic criteria in the differential diagnosis of brain tumours such as calcifications, cyst formation, punctuate or ring like enhancement, lipid contain, necrotic and haemorrhagic changes, it is still insufficient to give all needed information about the tumour nature. Still, there are few but important problems in the diagnosis of brain tumours including differentiation of the benign from the malignant tumours, the cystic/necrotic tumours from the brain abscess, the peritumoral oedema from the tumoral infiltration, the tumour recurrence from the radiation induced necrosis; identification of the exact tumour margins, predicting the tumour grade, and monitoring the response to treatment. Proton MRS can give different information about the tumour physiopathology including neuronal integrity, cell proliferation or degradation, energy metabolism and necrotic transformation of neuronal and tumoral tissue [2-7,9-11,14-16]. In this review, the diagnostic value of MRS added to MRI is evaluated.

\section{Methods}

MRS can be performed by using single or multi-voxel techniques or by using stimulated-echo acquisition mode (STEAM) or point-resolved excitation spin-echo (PRESS) sequences. Also MR spectra can be obtained by using long or short TE (time echo) according to T2 resonance of the desired metabolite. 
Comparison of single vs. multi-voxel techniques (Table 1) and STEAM vs. PRESS sequences (Table 2) gives us the opportunity to choose the appropriate technique due to relaxation properties of the desired metabolites. Typical PRESS selected MRS volumes are 50-150 cc with phase encode matrices of $8 \times 8 \times 8$ or $16 \times 8 \times 8$, nominal voxel sizes of $1-2 \mathrm{cc}$ with quadrature head coils or $0.2-0.5 \mathrm{cc}$ with surface coils at $1.5 \mathrm{~T}$ MR systems. Acquisition times for 3D chemical shift imaging (CSI) are kept to 12-17 min using TR $=1500 \mathrm{~ms}$ and TE $=40,144,270 \mathrm{~ms}$. Long TEs allow us to measure the major metabolites with long T2 resonance such as $n$-acetyl aspartate (NAA), choline (Cho), creatine and phosphocreatine $(\mathrm{Cr})$ and lactate, which are used as the main metabolites in tumour diagnosis. Shorter TEs, by inducing T2 losses and improving the SNR, also allow us to measure the metabolites with short T2 resonance such as lipid, alanine, glutamine and glutamate complex, glucose, myoinositol (mI) and other amino acids [4]. Among these short T2 metabolites, lipid, alanine and $\mathrm{mI}$ are very important in tumour diagnosis. For the MRS data, the first processing steps are to construct arrays of the spectra by applying time domain apodization, Fourier transformation (FT) and reconstructing the spatial dependence of the data. Whichever fitting algorithm is used, the number of spectra involved makes it critical that the procedure is fully automated, as well as robust to low SNR and missing peaks [14].

CSI has to be the choice of technique in tumour diagnosis, because simultaneous acquisition of spectra can be obtained from multiple regions and the quantification and metabolite maps can be created from CSI data. 2D FT and 3D FT implementations are possible. Although 2D MRS techniques support sufficient information within the chosen plane, 3D FT implementations give us the voluminous amounts of data, the exact definition the extent of metabolic abnormality outside the morphologic lesion and the functional metabolic data. By CSI techniques, 1-2 cc nominal voxel size can be obtained in $1.5 \mathrm{~T}$ and up to $0.4 \mathrm{cc}$ in $3 \mathrm{~T}$ static magnet systems [5,14]. Resolution limit increases from $0.5 \mathrm{cc}$ at $1.5 \mathrm{~T}$ to about $0.094 \mathrm{cc}$ at $3 \mathrm{~T}$ imagers [5,14], which causes a linear loss in SNR between $44 \%$ and $60 \%$ due to the decreasing line-width [5]. In combination of higher static magnetic fields of 3T, this enables high resolution CSI $(<0.5 \mathrm{cc})$ to be performed with satisfactory SNR in a reasonable time [5].

Table 1

Comparison of single vs. multi-voxel techniques

\begin{tabular}{ll}
\hline Single voxel & Multi voxel \\
\hline Easy to use & Hard to use \\
Shorter time & Longer time \\
Easy to interpret & Hard to interpret \\
More signal & Less signal \\
High spectral resolution & Low spectral resolution \\
Large voxel volume & Small voxel volume \\
No spatial resolution & High spatial resolution \\
Voxel placement critical & Allows metabolite \& quantification maps \\
\hline
\end{tabular}

Table 2

Comparison of STEAM vs. PRESS sequences

\begin{tabular}{ll}
\hline STEAM & PRESS \\
\hline Best at short TEs & Best at longer TEs \\
Little contamination & Larger contamination \\
Limited volumes & Larger volumes \\
Relatively low SNR & Retains full signal \\
Need more baseline corrections & More regular spectra \\
\hline
\end{tabular}


CSI has also had its own disadvantages. Pathological changes may occur only in a small fraction of the larger volume of interest (VOI) [5]. Metabolic changes may be masked or obscured in low resolution CSI, depending on the amount of healthy tissue or cerebrospinal fluid in the voxel [5]. An increase in SNR improves the spectral resolution, which provides better separation of metabolite signal, thereby improving the ability to identify and quantify chemical species [15].

Quantification is essential in MRS diagnosis. Different metabolite levels and ratios such as NAA/Cho, $\mathrm{NAA} / \mathrm{Cr}$, Cho/Cr, Lip/Cr, Lac/Cr can be obtained. Especially relative metabolite levels expressed as a percentage of tumour/contra lateral brain metabolite proportion are very useful in the comparison of the MR spectra obtained by different scan parameters or at different magnetic fields. It also allows us making the statistical analysis and comparison of MR spectra of different tumour types, when only a few standard spectra are available. It can also be used for assessing regression and progression of individual tumours [7].

Metabolic maps are displayed in a pseudo anatomic format rather than a spectrum. They provide the information in a more familiar way to radiologist and clinicians than the spectrum itself. Metabolic map imaging represents the distribution of particular species such as NAA, Cho, $\mathrm{Cr}$ and different ratio combinations. Images appear blurry due to low concentration of protons of desired species compared with water protons, but high-resolution techniques can improve the image quality.

\section{Differentiation of benign from malignant tumours}

On classical MR images, the definite and well-demarcated borders, homogeneous internal architecture, absence of enhancement or homogenous enhancement, small or absent peritumoral oedema and mass effect, absence of necrotic changes are the characteristic findings of benignity, but overlap of these features is not uncommon. Usually there is no major challenge for the diagnosis of high-grade malignant tumours; the differentiation of benign tumours from low-grade malignant ones is somewhat problematic. It is also difficult to differentiate pure haemorrhages from haemorrhagic tumours. Contrast enhancement is usually present in both subacute haemorrhagic infarcts and haemorrhagic tumours in the early phase and the differentiation of these two pathologies are only made by follow-up.

High Cho level is an excellent indicator of proliferative activity of neuroephitelial brain tumours and positively correlated with the cell density of the tumour [6]. MRS most probably detects the proliferating cells during $\mathrm{G}_{1}$ and S-phase [6]. Although there is a trend of higher mean Cho levels and lower mean NAA levels in higher-grade tumours, Cho levels are highly variable and there is also substantial overlap between different tumour grades $[2,14]$. Cho/NAA index can predict the tumour presence at a given location with $90 \%$ sensitivity and $86 \%$ specificity [9]. MRS is very effective in the differentiation of benign tumours from malignant ones (Figs 1-4). The most prominent MRS parameter is the decrease in NAA/Cho ratio [2]. This reduction is mainly due to both low NAA levels because of decrease in neuronal density and function, and high Cho levels because of increase in unbound Cho compounds due to increased membrane turnover. Low NAA/Cho ratio and presence of lactate peak is very typical for malignancy and lipid and lactate peaks are absent in all benign tumours [2,6]. Presence of lactate and lipid peaks are the indicator of increased necrosis and anaerobic glycolysis respectively and both can be present in both ischemia and tumours but, Cho level is high in tumours but low in ischemic lesions [11]. Whether haemorrhage is present or not, this high Cho signal indicates the presence of tumour [6]. Although decrease in apparent metabolite concentrations in necrotic glioblastoma multiformes (GBM) due to reduced cellular density has been previously reported [8], this reduction is not as the same as the absence of metabolites seen in ischemia. 


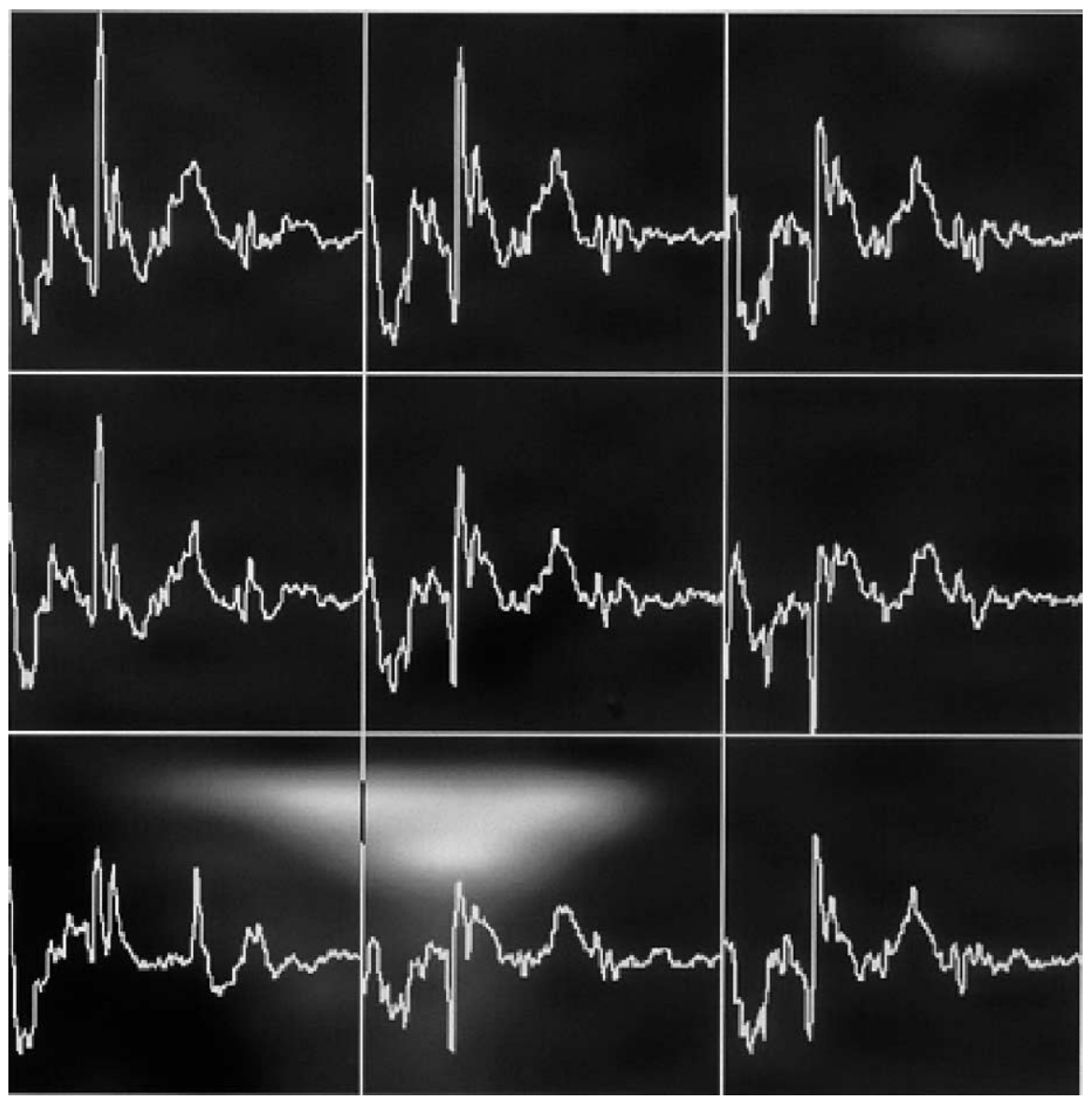

Fig. 1. 35 year-old male with intraventricular menengioma, multivoxel CSI images with TR $=1500 \mathrm{~ms}$ and TE $=40 \mathrm{~ms}$ show low NAA peak $(\delta=2.02 \mathrm{ppm})$ and high Cho mono-peak $(\delta=3.22 \mathrm{ppm})$ consistent with tumoral tissue. Alanine peak at $\delta=1.5 \mathrm{ppm}$ is characteristic for meningioma.

Alanine is significantly high to differentiate meningiomas from other benign and malignant tumours (Fig. 1), but the absence does not exclude meningiomas [2,11]. Although NAA has to be absent in meningiomas because of its non-neuronal origin, it can be detected possibly owing to contamination of the voxel with adjacent brain tissue or infiltrative nature of the tumour.

\section{Differentiation of cystic/necrotic tumours from brain abscess}

In immune compromised patients, incidence of both necrotic tumoral changes and abscess formation has increased. Differential diagnosis of these two entities is critical because they require different treatment protocols. Although hypointense ring seen on $\mathrm{T} 2 \mathrm{~W}$ images and regular ring-like enhancement are typical for abscess formation, these features can be overlap with necrotic tumours in MR images. In MRS spectra, abscess represents itself as with low Cho levels at $3.22 \mathrm{pm}$, moderate lipid levels at $1.3 \mathrm{ppm}$ [11] and high amino acid levels at $0.9 \mathrm{ppm}$ [4], but high Cho levels can easily differentiate it from abscess. Cho levels are statistically significantly lower than those of the contrast-enhancing neoplasm [11]. Abscesses have moderate lipid signal especially when originating from tuberculosis [2,11]. 


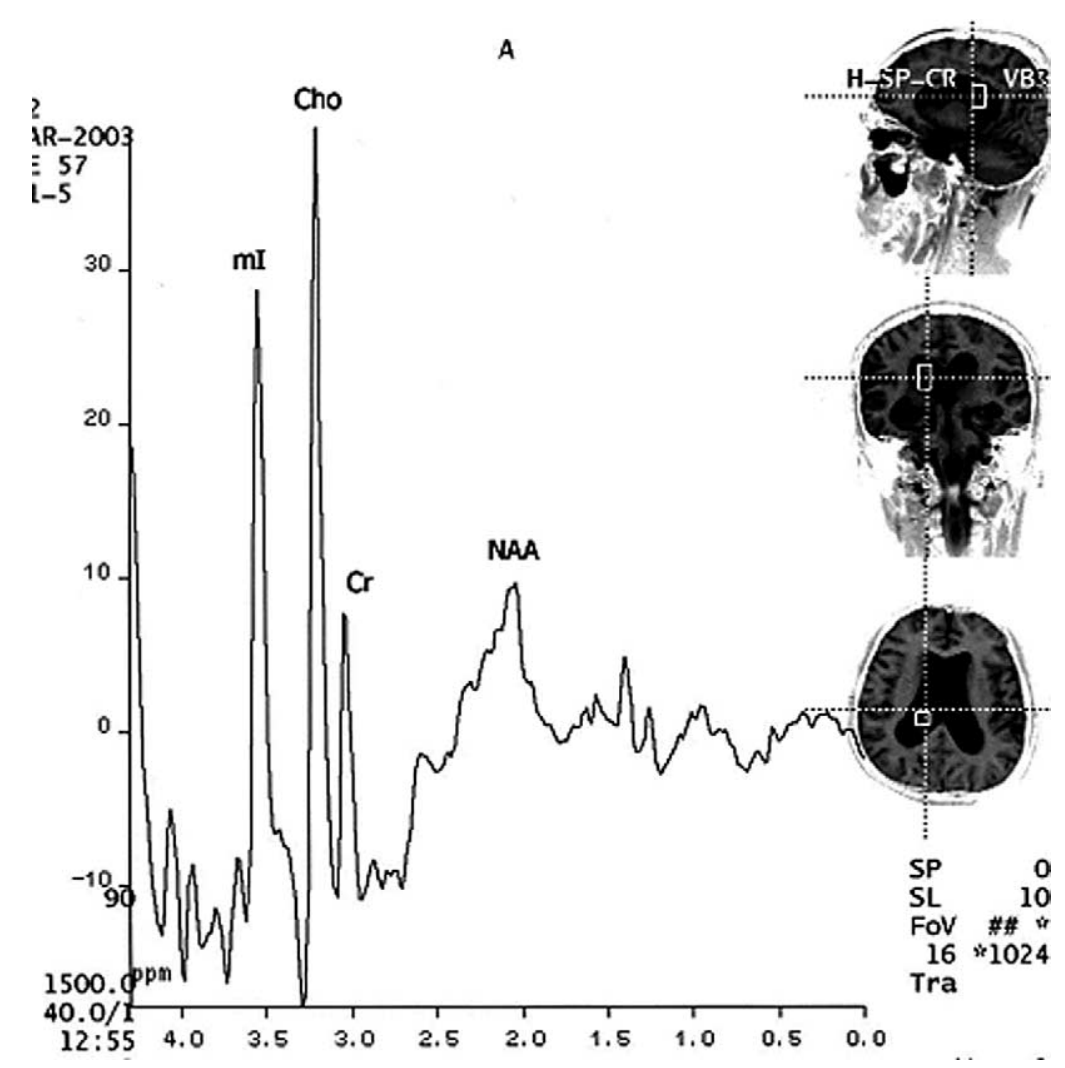

Fig. 2. 57 year-old man with intraventricular epandimoma. CSI spectrum $(\mathrm{TR} / \mathrm{TE}=1500 / 40 \mathrm{~ms})$ obtained from tumoral region shows low NAA $(\delta=2.02 \mathrm{ppm})$ and $\operatorname{Cr}(\delta=3.03 \mathrm{ppm})$ levels with high Cho $(\delta=3.22 \mathrm{ppm})$ and $\mathrm{mI}(\delta=3.6 \mathrm{ppm})$ peaks, which are consistent with low-grade tumour.

\section{Identification of exact tumour borders}

Peritumoral non-enhanced high signal intensity on T2 weighted images can represent the vasogenic oedema, the peritumoral infiltration or frequently both. Exact differentiation of this area is very critical for the exact treatment planning. Imperfect description of tumoral borders is one of the reasons of insufficient response treatment and leads to increase in tumour recurrence [14,15]. 36\% of high-grade tumours and $56 \%$ of lower grade tumours have spectral markers indicative of an active tumour outside regions of contrast enhancement [14]. By $0.5 \mathrm{cc}$ resolution limits in $1.5 \mathrm{~T}$ systems and $0.0094 \mathrm{cc}$ in 3T systems, multi-voxel MRS techniques can more clearly identify the tumoral borders than contrastenhanced images which lead to exact targeting for the treatment protocols and in choosing between local or more widespread therapy options $[5,14]$. Demonstration of tumoral spectra in non-enhanced peritumoral area is a good indicator of malignancy, but low spatial resolution of CSI is the only disadvantage of this technique. This has no effect on specificity, but lowers the sensitivity.

\section{Predicting the tumour grade}

One the most important problems encountering in brain tumour diagnosis is predicting the exact grade and type of viable tumoral tissue and the demonstration of tumoral heterogeneity between same grade 


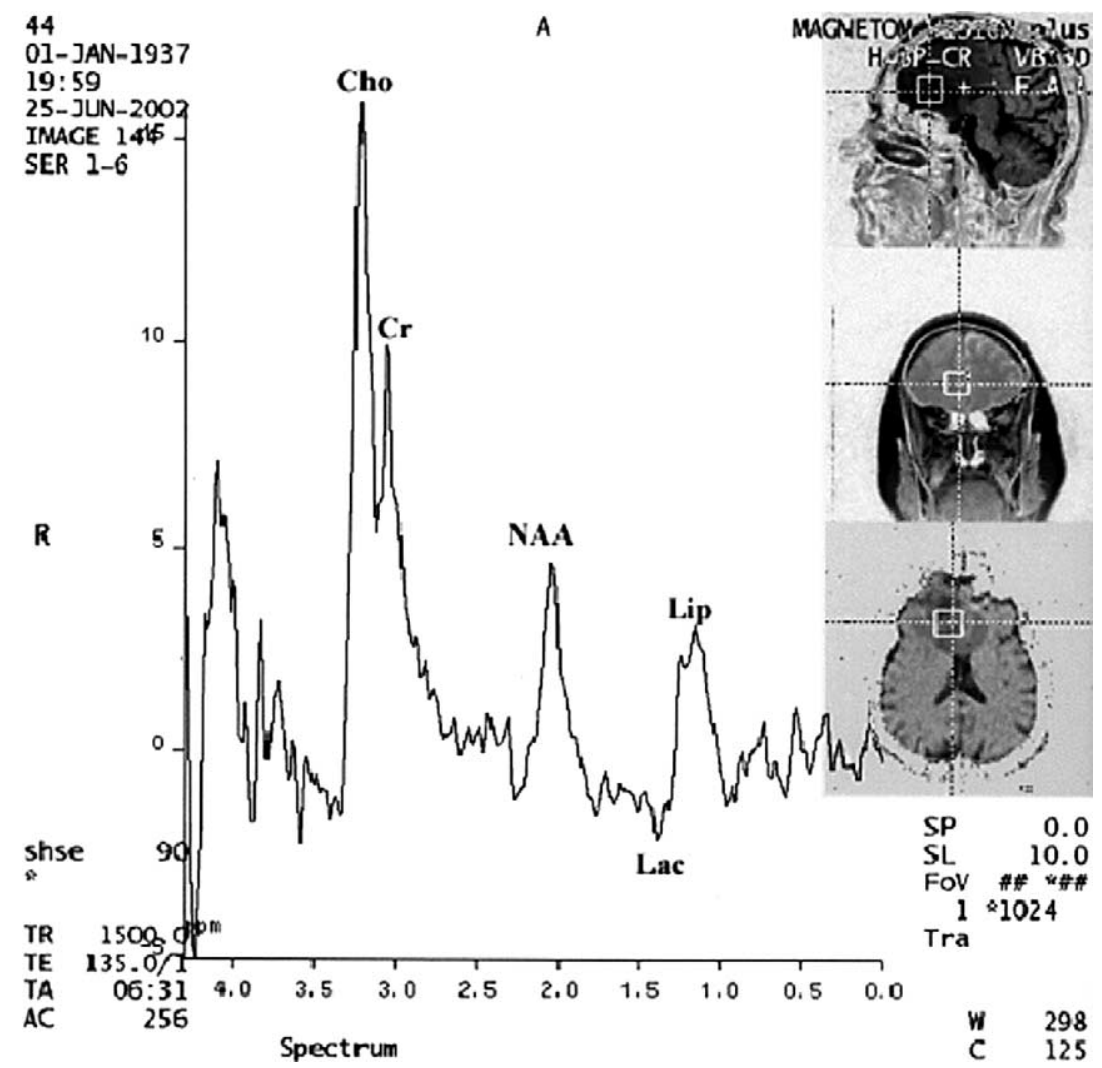

Fig. 3. 56 year-old male with anaplastic astrocytoma. Single voxel spectrum (TR/TE $=1500 / 135 \mathrm{~ms})$ shows prominent decrease in NAA/Cho ratio with low $\operatorname{Cr}(\delta=3.03 \mathrm{ppm})$ level. There are also prominent lactate or lipid $(\delta=1.1-1.6 \mathrm{ppm})$ peaks.

and type of tumours. Same tumour can have the areas with different grades and this usually represents borderline or mixed changes between different grades. Exact tumoral mapping is necessary for the exact treatment planning, which is as important as the identification of real tumoral borders.

Gadolinium chalets have long been used in tumour diagnosis, but contrast enhancement indicates the disruption of the blood-brain barrier rather than underlying regional vascularity. Also high-grade tumours have a higher percentage of contrast enhancing lesions than lower grade tumours, but areas of contrast enhancement are not always the most malignant portions of the tumour. On average, $1 / 3$ to $1 / 2$ of non-enhancing regions of malignant gliomas can contain active tumour that, if left untreated, may have a negative impact on survival [9]. Enhancing lesion represents partly to existence of active tumour tissue and partly to presence of treatment induced necrosis [14]. For these reasons contrast enhancement is not so effective in predicting the tumour grade.

Although high grade tumours had a higher percentage of contrast enhancing lesions than the lower grade tumours [14], tumour grading is not always related to the degree of enhancement, oedema or tumoral heterogeneity; the presence and extent of necrosis and cyst formation; the extension of the tumoral borders and the presence and the extent of haemorrhage or mass effect. This leads to insufficient grading of the brain tumours by the conventional MR images.

Although studies dealing with the effectiveness of MRS in the brain tumour grading gave us encouraging results, they also had some controversies. One of the MR spectroscopy parameters used in tumour 


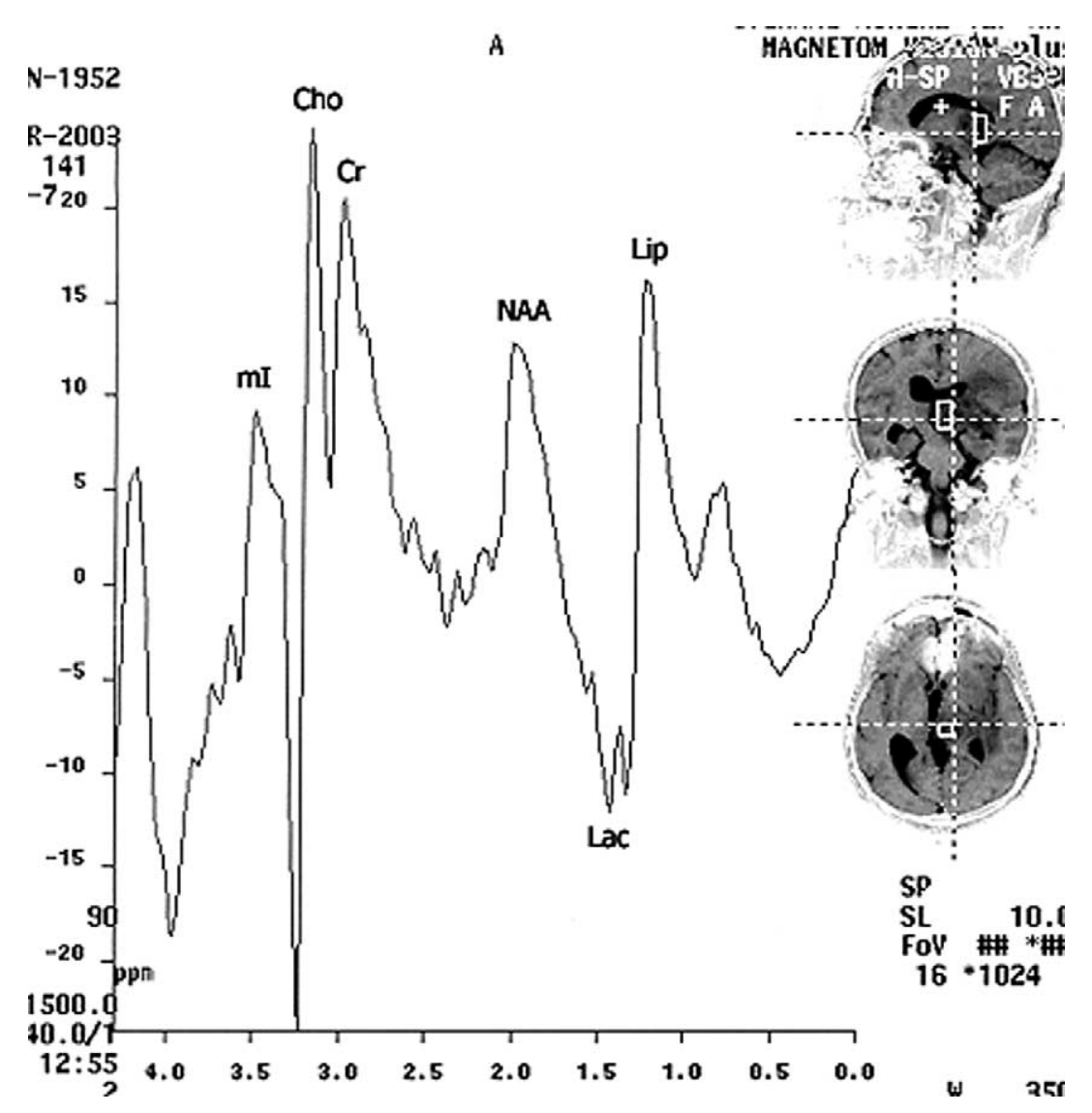

Fig. 4. 51 year-old man with lymphoma. CSI spectrum $(\mathrm{TR} / \mathrm{TE}=1500 / 40 \mathrm{~ms})$ obtained from tumoral region shows mild decrease in NAA/Cho ratio with normal $\operatorname{Cr}(\delta=3.03 \mathrm{ppm})$ and high $\mathrm{mI}(\delta=3.6 \mathrm{ppm})$ levels. Huge lipid peak is present at $\delta=1.1-1.6 \mathrm{ppm}$, which is characteristic for lymphoma.

grading is the decrease in NAA level. Reduction in NAA is mainly due to replacement of neurons by tumoral infiltration, which leads to decrease in function and density of the neurons. This decrease is moderate in low or mid-grade tumours and significant or absent in high-grade tumours [2]. NAA is also not present in metastasis and non-neuronal tumours such as meningiomas, which help us to differentiate primary tumours from metastasis and non-neuronal tumours. Sometimes these non-neuronal tumours can also have low NAA levels, which are probably due to contamination of the MRS voxel by normal brain parenchyma or due to infiltrative nature of these tumours, which leads to retention normal neuronal elements between tumour cells. So level of this decrease is not always well correlated with the degree of tumour grade.

Increase in Cho/Cr ratio is most prominent indicator of malignancy in MRS [2]. The long TE spectra are more clearly show this decrease than short TE ones [7]. This increase is mainly due to increase in Cho levels rather than a decrease in Cr levels. Cho compounds are increased because of increased membrane turnover and liberation of unbound Cho-containing compounds caused by the destruction of neurons during the malignant process. Especially phosphocholine and phosphoethanolamine show incremental increase in concentration during cellular proliferation, because of accelerated phosphorylation of Cho and ethanolamine or synthesis of membrane phospholipid precursors [6]. They can act as a long-term 
second messenger for cellular proliferation. Higher mean Cho levels are present in higher-grade tumours, but this trend is not always maintained in large necrotic/cystic GBMs, because of dilution of the apparent metabolite concentrations due to reduced cellular density [8].

Since Cr-bound phosphates are a substrate of the ADP/ATP cycle, $\mathrm{Cr}$ is considered to be an indicator of energy metabolism and it is quite constant in various metabolic conditions, which leads to be used as an internal standard for semi-quantitative evaluation of metabolic changes of other neuronal metabolites [12]. Cr is low in all tumours when compared with normal groups, but there is no significant difference between different tumour grades [2].

Lipid peaks are more frequent in higher-grade tumours and well correlate with the extent of cell necrosis, but their absence does not exclude them [7]. Mobile lipids are also observed to arise in tumour cells under hypoxic stress prior to necrosis [1]. Hypoxic stress likely occurs following malignant transformation when there are many rapidly dividing cells but not an adequate blood supply [1]. An increase in mobile lipid signals may be an early marker of malignant transformation prior to necrosis [1], but some benign and malign lesions such as tuberculosis granulomas and lymphomas may have high lipid levels independently from tumour grade $[2,17]$. The highest lipid concentrations were observed in metastasis [2]. The average lipid signal in long-TE spectra was higher in metastasis than GBM, possibly indicating a difference in T2 relaxation times [1]. Enormously increased lipid peak differentiated lymphomas from all other lesions (Fig. 4) [17].

Lactate is produced by anaerobic glycolysis in tumours with hypoxia due to a mismatch between glycolysis and oxygen supply and tends to be higher in high-grade tumours. Both Lip and Lac concentrations can be lower in GBM than in grade II and III astrocytomas because of dilution of metabolite concentrations due to aggressive necrosis [7]. There is a poor correlation between lactate concentrations with tumour grade, due to its levels being dependent on the balance between production and clearance [1].

Levels of $\mathrm{mI} / \mathrm{Cr}$ were higher $(0.82 \pm 0.25)$ in patients with low-grade astrocytoma (Fig. 2$)$, intermediate $(0.49 \pm 0.07)$ in control subjects, lower in patients with anaplastic astrocytoma $(0.33 \pm 0.16)$ and $\operatorname{GBM}(0.15 \pm 0.12)[3]$.

Metabolic data have large variations and overlaps in grading due to the nonuniformity of localisation techniques, the variable attempts to avoid contamination of spectra by metabolites from the regions of oedema or tumoral infiltration, and the usage of different acquisition parameters. At TE $=135 \mathrm{~ms}$, mobile lipid signals are only observed in $41 \%$ of high grade tumours [12], and any lactate present is in opposite phase to lipid signals; hence T2 losses or signal cancellations may mask the true correlation with necrosis and hypoxia [1]. Mixed neoplasm result in intratumoral regions composed of various amounts of normal brain, inflammatory tissue, necrosis and active tumour. Since tumour growth is not well regulated, variations in cellular metabolism and cell density also occur. Individual tumour spectra are thus the average of several different tissue types and inherently variable [7]. MRS is very useful but still insufficient to show tumoral heterogeneity because of lower spatial resolution. Even if the spectroscopic ROI appears to be homogenous and without partial volume contamination either from surrounding brain parenchyma or regressive events such as necrotic or cystic transformation, this cannot be excluded for microscopic MR-invisible necroses or cysts [6].

\section{Monitoring the response to treatment}

Abnormal high Cho concentrations can be an early sign of malignant transformation. Tumour represents itself by high Cho levels with high $\mathrm{Cho} / \mathrm{Cr}$ ratio greater than 1.3 [15]. On the other hand necrosis 


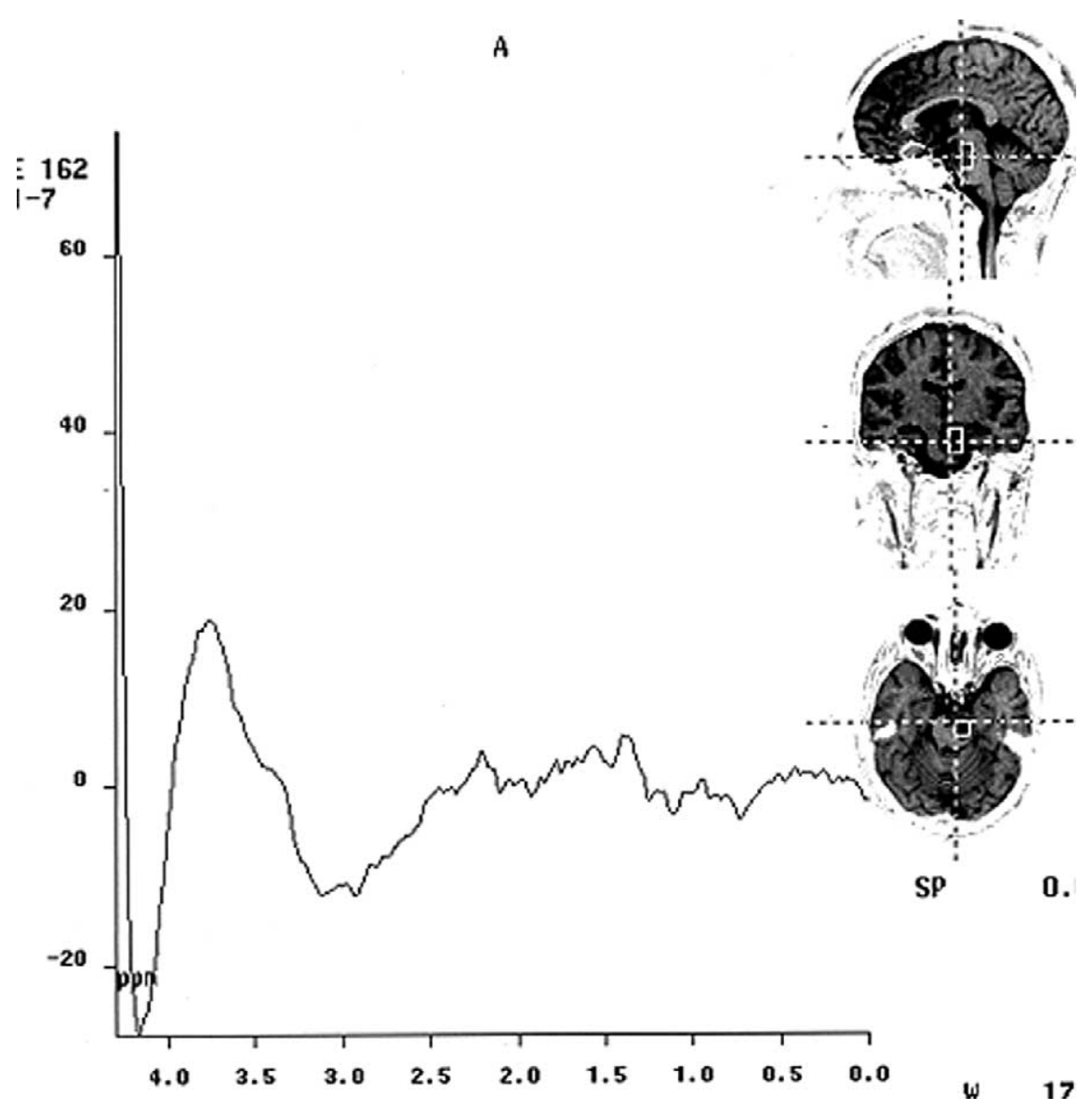

Fig. 5. 28 year-old female with pontin glioma after radiotherapy. CSI spectrum (TR/TE $=1500 / 40 \mathrm{~ms}$ ) obtained from tumoral region shows no major metabolite peaks, which is consistent with radiation necrosis.

has demonstrated by very low values of all metabolites (Fig. 5). Cho/Cr ratio appears to be the best parameter for determining whether a lesion contains predominantly tumour $(>1.3)$ or radiation necrosis $(<1.3)$ [15]. Significant reduction of Cho levels after therapy indicating the transformation of tumour to necrotic tissue [14]. MRS is also valuable in defining the therapy target volume [14]. Progressive tumours show a Cho increase between serial studies of more than $45 \%$, whereas stable cases show an elevation less than $35 \%$ or even decreased Cho signal intensity [16]. Regression of tumoral load is consistent with decrease in Cho concentrations.

\section{Conclusion}

Multi-voxel 2D or 3D CSI and metabolic map imaging has to be the choice of study to evaluate brain tumours, because it provides simultaneous acquisition of spectra from multiple regions with voxel volume as small as 1-2 cc. Especially metabolic mapping leads to exact definition of the metabolic abnormality in and outside the morphologic lesion. 3D implementations can obtain more voluminous data from the other planes outside the interested slice and lead us to exact delineation of the tumour borders. 
MRS is more definitive then FDG-PET in identifying the tumours [13]. The percentage of correct diagnosis can be increased from $55.1 \%$ to $70.5 \%$ and the percentage of incorrect diagnosis can be lowered from $15.3 \%$ to $9.1 \%$ by the addition of MRS to MRI in tumour diagnosis [11]. MRS results are very specific but less sensitive in tumour diagnosis because of low spatial resolution of MRS techniques. Small percentage of tumour in a big voxel filled with normal brain parenchyma can lead to these false negative results. Systems with higher spatial and temporal resolution are needed to obtain sufficient information to understand the exact nature of the tumour and the morphological and physiological changes occur in the surrounding brain parenchyma. Because both spatial resolution and SNR depend linearly to magnetic field, these problems can overcome at higher magnetic field strengths. By the advent of scanner systems with higher static magnetic fields and faster imaging techniques such parallel imaging, which give us the opportunity of collecting functional data such as tumoral response to hypoxic stress, can improve the diagnostic effectiveness of MRS in tumour diagnosis and grading. Current MRS studies provide the discrimination of solid tumour, viable brain, and necrotic tissues based on cellular metabolite levels. Especially combining with the perfusion and functional MR techniques, MR images accompanied by MRS techniques will allow us to assess the whole tumoral morphologic and hemodynamic changes, and to monitor the response of the tumour and surrounding tissue to treatment.

\section{References}

[1] I. Barba, M.E. Cabanas and C. Arus, The relationship between nuclear magnetic resonance-visible lipids, lipid droplets, and cell proliferation in cultured C6 cells, Cancer Res. 59 (1999), 1861-1868.

[2] N. Bulakbasi, M. Kocaoglu, F. Ors, C. Tayfun and T. Ucoz, Combination of single voxel proton MR spectroscopy and apparent diffusion coefficient calculation in the evaluation of common brain tumors, AJNR. Am. J. Neuroradiol. 23 (2003), 225-233.

[3] M. Castillo, J.K. Smith and L. Kwock, Correlation of myo-inositol levels and grading of cerebral astrocytomas, AJNR Am. J. Neuroradiol. 21 (2000), 1645-1649.

[4] S. Grand, G. Passaro, A. Ziegler, F. Esteve, C. Boujet, D. Hoffmann, C. Rubin, C. Segebarth, M. Decorps, J.F. Le Bas and C. Remy, Necrotic tumor versus brain abscess: importance of amino acids detected at $1 \mathrm{H}$ MR spectroscopy - initial results, Radiology 213 (1999), 785-793.

[5] S. Gruber, V. Mlynarik and E. Moser, High resolution 3D proton spectroscopic imaging of the human brain in 3T: SNR issues and application for anatomy-matched voxel sizes, Mag. Reson. Med. 49 (2003), 299-306.

[6] S. Herminghaus, U. Pilatus, W. Moller-Hartmann, P. Raab, H. Lanfermann, W. Schlote and F.E. Zanella, Increased choline levels coincide with enhanced proliferative activity of human neuroepithelial brain tumors, NMR Biomed. 15 (2002), 385392.

[7] F.A. Howe, S.J. Barton, S.A. Cudlip, M. Stubbs, D.E. Saunders, M. Murphy, P. Wilkins, K.S. Opstad, V.L. Doyle, M.A. McLean, B.A. Bell and J.R. Griffiths, Metabolic profiles of human brain tumors using quantitative in vivo ${ }^{1} \mathrm{H}$ magnetic resonance spectroscopy, Magn. Reson. Med. 49 (2003), 223-232.

[8] Y. Kinoshita and A. Yokota, Absolute concentrations of metabolites in human brain tumors using in vitro proton magnetic resonance spectroscopy, NMR Biomed. 10 (1997), 2-12.

[9] T.R. McKnight, M.H. von dem Bussche, D.B. Vigneron, Y. Lu, M.S. Berger, M.W. McDermott, W.P. Dillon, E.E. Graves, A. Pirzkall and S.J. Nelson, Histopathological validation of a three-dimensional magnetic resonance spectroscopy index as a predictor of tumor presence, J. Neurosurg. 97 (2002), 794-802.

[10] B.L. Miller, A review of chemical issues in 1H-MRS spectroscopy: N-acetyl-L-aspartate, creatine and choline, NMR Biomed. 4 (1999), 47-52.

[11] W. Moller-Hartmann, S. Herminghaus, T. Krings, G. Marquardt, H. Lanfermann, U. Pilatus and F.E. Zanella, Clinical application of proton magnetic resonance spectroscopy in the diagnosis of intracranial mass lesions, Neuroradiology 44 (2002), 371-381.

[12] W.G. Negendank, R. Sauter, T.R. Brown, J.L. Evelhoch, A. Falini, E.D. Gotsis, A. Heerschap, K. Kamada, B.C. Lee, M.M. Mengeot, E. Moser, K.A. Padavic-Shaller, J.A. Sanders, T.A. Spraggins, A.E. Stillman, B. Terwey, T.J. Vogl, K. Wicklow and R.A. Zimmerman, Proton magnetic resonance spectroscopy in patients with glial tumors: a multicenter study, J. Neurosurg. 84 (1996), 449-458. 
[13] S.J. Nelson, M.R. Day, P.J. Buffone, L.L. Wald, T.F. Budinger, R. Hawkins, W.P. Dillon, S. Huhn, M.D. Prados, S. Chang and D.B. Vigneron, Alignment of volume MR images and high resolution [18F] fluorodeoxyglucose PET images for the evaluation of patients with brain tumors, J. Comput. Assist. Tomogr. 21 (1997), 183-191.

[14] S.J. Nelson, D.B. Vigneron and W.P. Dillon, Serial evaluation of patients with brain tumors using volume MRI and $3 \mathrm{D}{ }^{1} \mathrm{H}$ MRSI, NMR Biomed. 12 (1999), 123-128.

[15] J.D. Rabinov, P.L. Lee, F.G. Barker, D.N. Louis, G.R. Harsh, G.R. Cosgrove, E.A. Chiocca, A.F. Thornton, J.S. Loeffler, J.W. Henson and R.G. Gonzalez, In vivo 3-T MR spectroscopy in the distinction of recurrent glioma versus radiation effects: Initial experience, Radiology 225 (2002), 871-879.

[16] G. Tedeschi, N. Lundbom, R. Raman, S. Bonavita, J.H. Duyn, J.R. Alger and G. Di Chiro, Increased choline signal coinciding with malignant degeneration of cerebral gliomas: a serial proton magnetic resonance spectroscopy imaging study, J. Neurosurg. 87 (1997), 516-524.

[17] M. Utriainen, M. Komu, V. Vuorinen, P. Lehikoinen, P. Sonninen, T. Kurki, T. Utriainen, A. Roivainen, H. Kalimo and H. Minn, Evaluation of brain tumor metabolism with [11C]choline PET and 1H-MRS, J. Neurooncol. 62 (2003), 329-338. 


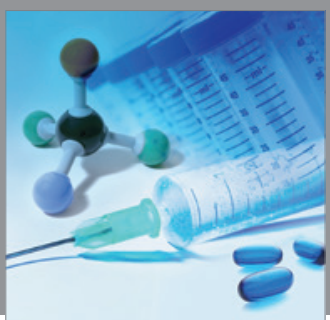

International Journal of

Medicinal Chemistry

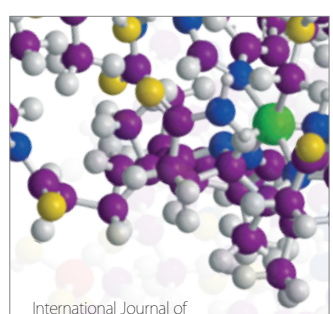

Carbohydrate Chemistry

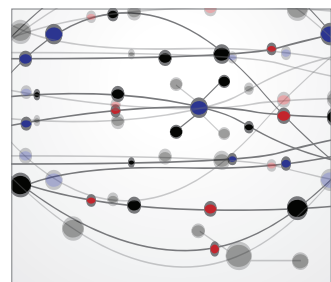

The Scientific World Journal
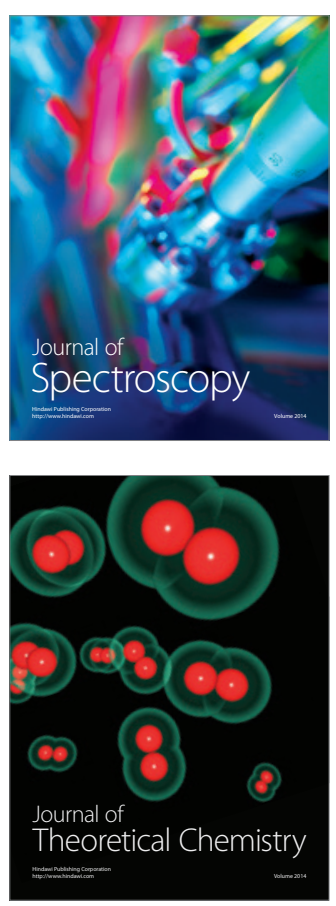
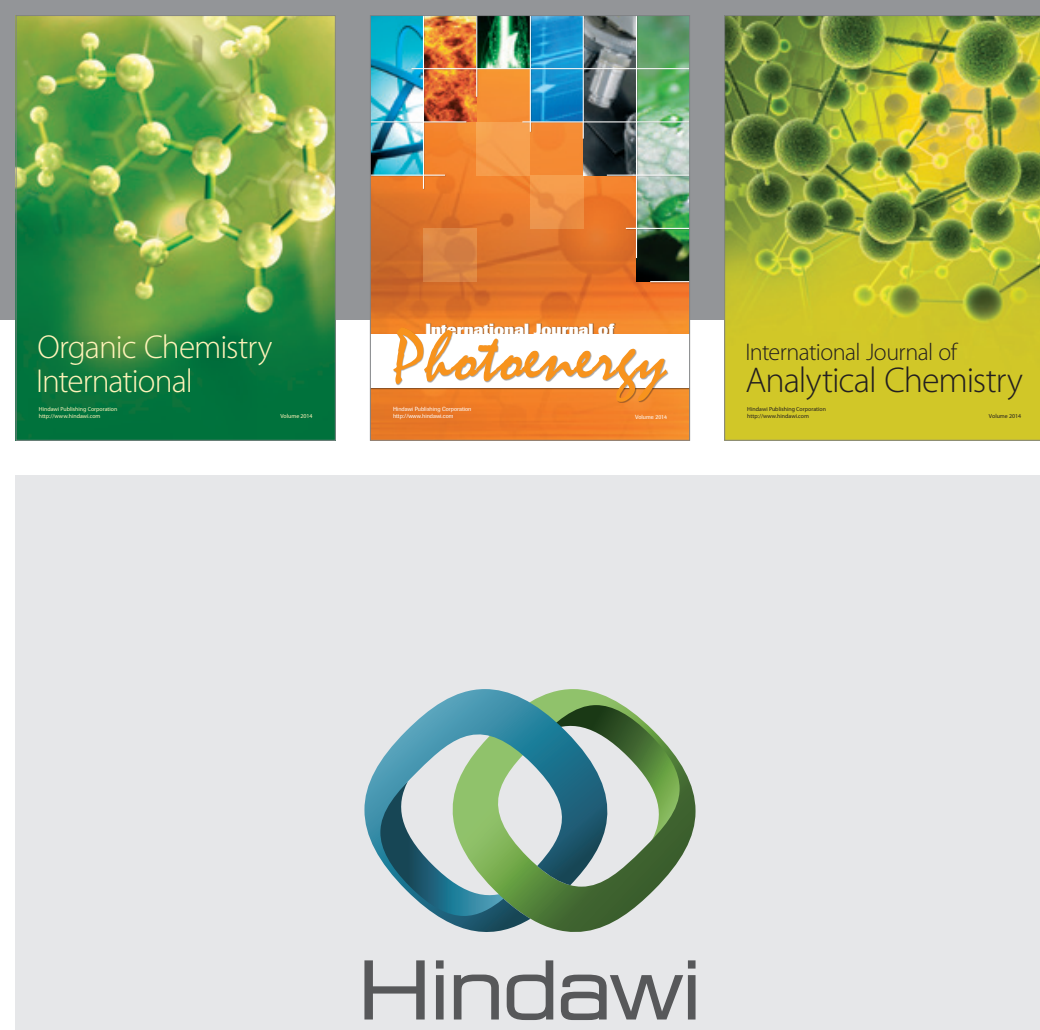

Submit your manuscripts at

http://www.hindawi.com
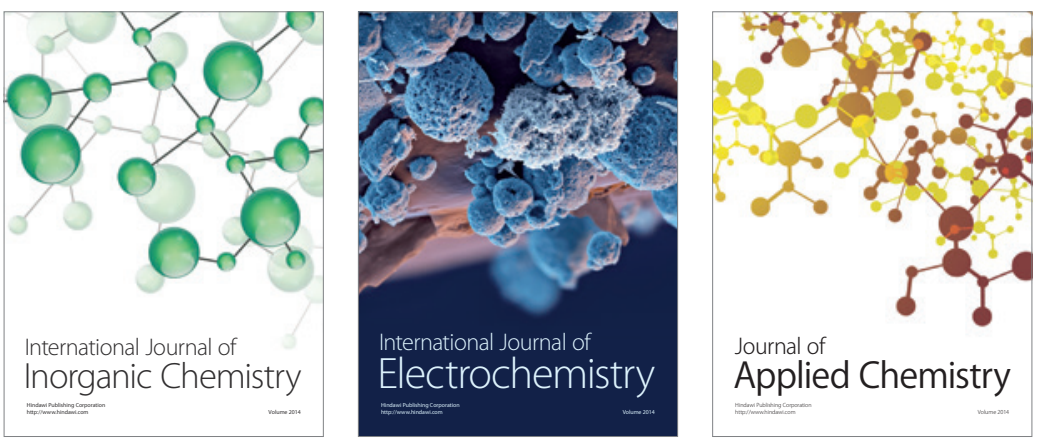

Journal of

Applied Chemistry
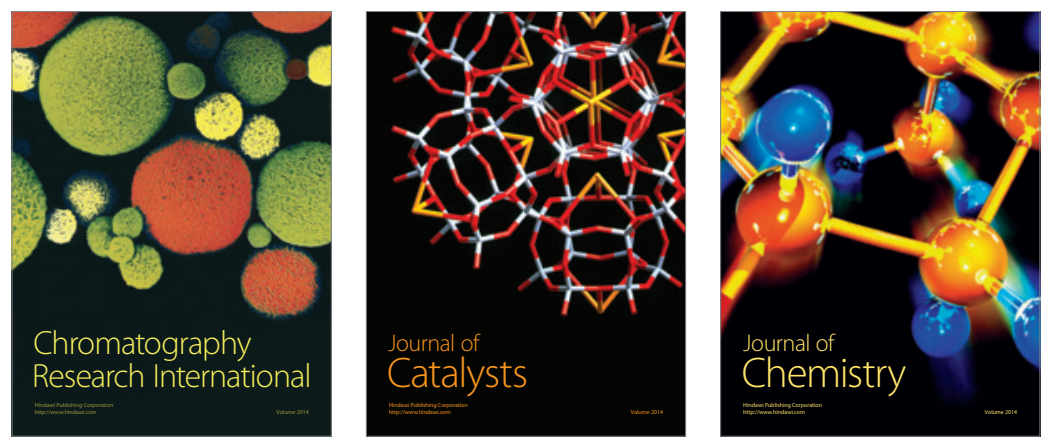
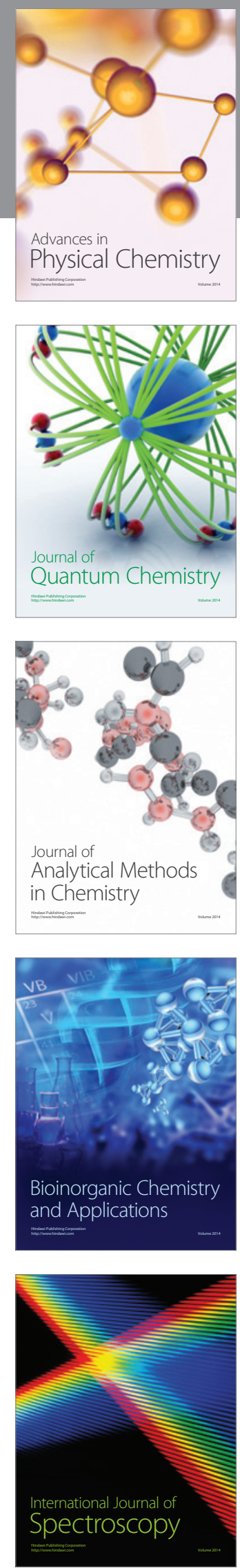